\title{
Simultaneous Controllability for a System with Resistance Term
}

G.O. ANTUNES, F.A. ARARUNA, L.A. MEDEIROS ${ }^{1}$, Instituto de Matemática, UFRJ, Caixa Postal 68530, 21945-970 Rio de Janeiro, RJ, Brasil.

\begin{abstract}
In this work we study the simultaneous controllability for a system of equations that constitutes a model of dynamical elasticity for incompressible materials.
\end{abstract}

\section{Introduction}

Let $\Omega$ be a bounded domain of $\mathbb{R}^{n}$ with regular boundary $\Gamma$. Let $Q$ denote the cylinder $\Omega \times(0, T)$ whose lateral boundary is given by $\Sigma=\Gamma \times(0, T)$.

In this work, we shall consider the simultaneous controllability for the system

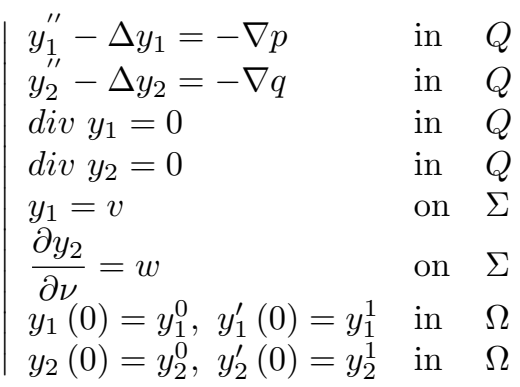

where $p=p(x, t)$ and $q=q(x, t)$ denote the resistance terms.

Physically the above system models the small deformations or displacements of the solid body $\Omega \subset \mathbb{R}^{n}$ composed of incompressible elastic materials, subject to controls acting on the boundary $\Sigma$.

The simultaneous controllability for the system (1.1) is formulated as follows: given $T>0$ large enough, find a Hilbert space $H$ such that for every $\operatorname{set}\left\{y_{1}^{0}, y_{1}^{1}, y_{2}^{0}, y_{2}^{1}\right\}$ belonging to $H$, there exists a pair of controls $\{v, w\}$, such that a solution $\left\{y_{1}(v), y_{2}(w)\right\}$ of (1.1) satisfies the equilibrium condition

$$
y_{1}(T)=y_{1}^{\prime}(T)=y_{2}(T)=y_{2}^{\prime}(T)=0,
$$

and

$$
w=\frac{\partial v}{\partial t} \quad \text { on } \quad \Sigma .
$$

\footnotetext{
${ }^{1}$ Partially supported by PCI-LNCC-MCT-2001.
} 
We investigate this problem by means of the Hilbert Uniqueness Method (HUM) idealized by Lions [6].

The problem of the simultaneous controllability was initially studied by Lions [6]. Kapitonov [4] investigated a similar question. For exact controllability we mention Cavalcanti et al [2].

\section{Notations, Assumptions and Results}

We consider $\Omega_{0}, \Omega_{1} \subset \mathbb{R}^{n}, n \geq 2$, two bounded domains with boundary $\partial \Omega_{0}, \partial \Omega_{1}$ of class $C^{2}$, such that

$$
\bar{\Omega}_{1} \subset \Omega_{0},
$$

$\Omega_{0}, \Omega_{1}$ are star shaped with respect to $x_{0} \in \bar{\Omega}_{1}$.

Let us assume

$$
\Omega=\Omega_{0} \backslash \bar{\Omega}_{1} .
$$

We set $m(x)=x-x_{0}, R\left(x_{0}\right)=\max _{x \in \bar{\Omega}}|m(x)|$ and define

$$
\Gamma\left(x_{0}\right)=\{x \in \Gamma ; m(x) \cdot \nu(x)>0\} \quad \text { and } \quad \Gamma_{*}\left(x_{0}\right)=\Gamma \backslash \Gamma\left(x_{0}\right) .
$$

The following partition of the boundary is chosen

$$
\Gamma_{0}=\Gamma\left(x_{0}\right), \quad \Gamma_{1}=\Gamma_{*}\left(x_{0}\right) .
$$

The action in the boundary $\Sigma$ is assumed to be of the following type

$$
\begin{aligned}
& y_{1}=\left\{\begin{array}{lll}
v & \text { on } & \Sigma_{0}=\Gamma_{0} \times(0, T) \\
0 & \text { on } & \Sigma \backslash \Sigma_{0}=\Gamma_{1} \times(0, T)
\end{array},\right. \\
& \frac{\partial y_{2}}{\partial \nu}=w \quad \text { on } \quad \Sigma_{0}, \\
& y_{2}=0 \quad \text { on } \quad \Sigma \backslash \Sigma_{0} \text {. }
\end{aligned}
$$

In addition we consider the following hypotheses

$$
p=q=0 \quad \text { on } \quad \Sigma_{0} .
$$

We introduce the following Hilbert spaces

$$
\begin{gathered}
V=\left\{u \in\left(H_{0}^{1}(\Omega)\right)^{n} ; \operatorname{div} u=0\right\}, \\
H=\left\{u \in\left(L^{2}(\Omega)\right)^{n} ; \operatorname{div} u=0, u \cdot \eta=0 \text { on } \Gamma\right\},
\end{gathered}
$$

with the structure of internal product and norm induced by $\left(H_{0}^{1}(\Omega)\right)^{n}$ and $\left(L^{2}(\Omega)\right)^{n}$, respectively. We still consider

$$
\mathcal{V}=\left\{\varphi \in(D(\Omega))^{n} ; \operatorname{div} \varphi=0\right\},
$$




$$
X=\left\{\varphi \in\left(H^{1}(\Omega)\right)^{n} ; \operatorname{div} \varphi=0, \varphi=0 \text { on } \Gamma_{1}\right\}
$$

and

$$
Y=\left\{\varphi \in X ; \Delta \varphi \in\left(L^{2}(\Omega)\right)^{n}, \frac{\partial \varphi}{\partial \nu}=0 \text { on } \Gamma_{0}\right\} .
$$

The energy associated with the system (1.1) is given by

$$
E(t)=E_{1}(t)+E_{2}(t),
$$

where

$$
E_{i}(t)=\frac{1}{2}\left\{\sum_{k=1}^{n} \int_{\Omega}\left|\nabla y_{k i}(t)\right|^{2} d x+\sum_{k=1}^{n} \int_{\Omega}\left|y_{k i}^{\prime}(t)\right|^{2} d x\right\}, \quad i=1,2 .
$$

\section{Inverse Inequality}

Let us consider the following problem

$$
\mid \begin{array}{lll}
\Phi_{1}^{\prime \prime}-\Delta \Phi_{1}=-\nabla p & \text { in } & Q \\
\operatorname{div} \Phi_{1}=0 & \text { in } & Q \\
\Phi_{1}=0 & \text { on } & \Sigma \\
\Phi_{1}(0)=\Phi_{1}^{0}, \Phi_{1}^{\prime}(0)=\Phi_{1}^{1} & \text { in } & \Omega .
\end{array}
$$

Lions in [7] showed that the solution $\Phi_{1}$ of (3.1) has the hidden regularity $\frac{\partial u}{\partial \nu} \in$ $\left(L^{2}(\Sigma)\right)^{n}$ and that mapping

$$
\left\{\Phi_{1}^{0}, \Phi_{1}^{1}\right\} \mapsto \frac{\partial u}{\partial \nu}
$$

is continuous from $V \times H$ in $\left(L^{2}(\Sigma)\right)^{n}$.

Remark 3.1: Multiplying the equation in $(3.1)_{1}$ by $m \nabla \Phi_{1}$ and integrating in $Q$,

$$
\int_{Q} \Phi_{1}^{\prime \prime} m \nabla y_{1} d x d t-\int_{Q} \Delta \Phi_{1} m \nabla \Phi_{1} d x d t=\int_{Q}(-\nabla p) m \nabla \Phi_{1} d x d t
$$

Let us put

$$
X=-\int_{Q} \frac{\partial p}{\partial x_{i}} m_{k} \frac{\partial \Phi_{1 i}}{\partial x_{k}} d x d t,
$$

with the summation convention of repeated indices. Integrating by parts in $x_{k}$ and observing $(3.1)_{3}$ comes that

$$
\begin{aligned}
X & =-\left.\int_{0}^{T} \frac{\partial p}{\partial x_{i}} m_{k} \Phi_{1 i} d t\right|_{\Gamma}+\int_{Q} \frac{\partial}{\partial x_{k}}\left(\frac{\partial p}{\partial x_{i}} m_{k}\right) \Phi_{1 i} d x d t \\
& =\int_{Q} \frac{\partial^{2} p}{\partial x_{k} \partial x_{i}} m_{k} \Phi_{1 i} d x d t+\int_{Q} \frac{\partial p}{\partial x_{i}} \frac{\partial m_{k}}{\partial x_{k}} \Phi_{1 i} d x d t \\
& =\int_{Q} \frac{\partial^{2} p}{\partial x_{k} \partial x_{i}} m_{k} \Phi_{1 i} d x d t+n \int_{Q} \frac{\partial p}{\partial x_{i}} \Phi_{1 i} d x d t .
\end{aligned}
$$


Now,

$$
n \int_{Q} \frac{\partial p}{\partial x_{i}} \Phi_{1 i} d x d t=\left.n \int_{0}^{T} p \Phi_{1 i} d t\right|_{\Gamma}-n \int_{Q} p \frac{\partial \Phi_{1 i}}{\partial x_{i}} d x d t=-n \int_{Q} p d i v \Phi_{1} d x d t=0 .
$$

Therefore

$$
X=\int_{Q} \frac{\partial^{2} p}{\partial x_{k} \partial x_{i}} m_{k} \Phi_{1 i} d x d t .
$$

Making integration by parts again, it results in

$$
\begin{aligned}
X & =\left.\int_{0}^{T} \frac{\partial p}{\partial x_{k}} m_{k} \Phi_{1 i} d t\right|_{\Gamma}-\int_{Q} \frac{\partial p}{\partial x_{k}} \frac{\partial}{\partial x_{i}}\left(m_{k} \Phi_{1 i}\right) d x d t \\
& =-\int_{Q} \frac{\partial p}{\partial x_{k}} \frac{\partial m_{k}}{\partial x_{i}} \Phi_{1 i} d x d t-\int_{Q} \frac{\partial p}{\partial x_{k}} m_{k} \frac{\partial \Phi_{1 i}}{\partial x_{i}} d x d t \\
& =-\int_{Q} \frac{\partial p}{\partial x_{k}} \delta_{i}^{k} \Phi_{1 i} d x d t-\int_{Q} \frac{\partial p}{\partial x_{k}} m_{k} d i v \Phi_{1} d x d t \\
& =\int_{Q} p \operatorname{div} \Phi_{1} d x d t-\int_{Q} \frac{\partial p}{\partial x_{k}} m_{k} d i v \Phi_{1} d x d t=0 .
\end{aligned}
$$

Lemma 3.1. Assume $q=q(x) \in\left[C^{1}(\bar{\Omega})\right]^{n}$. Then, for every solution of (3.1) with data $\left\{\Phi_{1}^{0}, \Phi_{1}^{1}\right\} \in V \times H$, the following identity holds:

$$
\begin{aligned}
& \frac{1}{2} \sum_{k=1}^{n} \int_{\Sigma} q_{k}(x) \cdot \nu_{k}(x)\left|\frac{\partial \Phi_{1}}{\partial \nu}\right|^{2} d \Sigma \\
& =\left.\left(\Phi_{1}^{\prime}(t), q(x) \nabla \Phi_{1}\right)\right|_{0} ^{T} d x+\sum_{k=1}^{n} \int_{Q} \frac{\partial p}{\partial x_{i}} q_{k} \frac{\partial \Phi_{1 i}}{\partial x_{k}} d x d t \\
& \quad+\frac{1}{2} \sum_{k=1}^{n} \int_{Q} \frac{\partial q_{k}}{\partial x_{k}}\left(\left|\Phi_{1}^{\prime}(x, t)\right|^{2}-\left|\nabla \Phi_{1}(x, t)\right|^{2}\right) d x d t+\sum_{j, k=1}^{n} \int_{Q} \frac{\partial q_{k}}{\partial x_{j}} \frac{\partial \Phi_{1 i}}{\partial x_{k}} \frac{\partial \Phi_{1 i}}{\partial x_{j}} d x d t .
\end{aligned}
$$

Lemma 3.2. Assume $T>2 R\left(x_{0}\right)$. Then the following estimate holds for every solution of (3.1) with data $\left\{\Phi_{1}^{0}, \Phi_{1}^{1}\right\} \in V \times H$,

$$
E_{01} \leq \frac{R\left(x_{0}\right)}{2\left(T-2 R\left(x_{0}\right)\right)} \int_{\Sigma_{0}}\left|\frac{\partial \Phi_{1}}{\partial \nu}\right|^{2} d \Sigma .
$$

In the proof of the Lemmas 3.1 and 3.2 we used the idea of Lions [6] together with the Remark 3.1.

We consider now the homogeneous problem for $\Phi_{2}$

$$
\begin{array}{lll}
\Phi_{2}^{\prime \prime}-\Delta \Phi_{2}=-\nabla q & \text { in } & Q \\
\text { div } \Phi_{2}=0 & \text { in } & Q \\
\frac{\partial \Phi_{2}}{\partial \nu}=0 & \text { on } & \Sigma_{0} \\
\Phi_{2}=0 & \text { on } & \Sigma_{1} \\
\Phi_{2}(0)=\Phi_{2}^{0}, \Phi_{2}^{\prime}(0)=\Phi_{2}^{1} & \text { in } & \Omega .
\end{array}
$$


Similarly to the Remark 3.1, multiplying the equation in (3.4) $)_{1}$ by $m \nabla \Phi_{2}$ and integrating in $Q$, we obtain

$$
\int_{Q} \Phi_{2}^{\prime \prime} m \nabla \Phi_{2} d x d t-\int_{Q} \Delta \Phi_{2} m \nabla \Phi_{2} d x d t=\int_{Q}(-\nabla q) m \nabla \Phi_{2} d x d t=0 .
$$

Lemma 3.3. Assume $q \in\left[W^{1, \infty}(\Omega)\right]^{n}$. Then for every weak solution of the homogeneous problem (3.4) with data $\left\{\Phi_{2}^{0}, \Phi_{2}^{1}\right\} \in Y \times X$ the following identity holds

$$
\begin{aligned}
\int_{\Sigma_{0}} q_{k} \nu_{k}\left(\left|\Phi_{2}^{\prime}\right|^{2}-\right. & \left.\left|\nabla_{\sigma} \Phi_{2}\right|^{2}\right) d \Sigma+\frac{1}{2} \int_{\Sigma_{1}} q_{k} \nu_{k}\left|\frac{\partial \Phi_{2}}{\partial \nu}\right|^{2} d \Sigma \\
= & \left.\left(\Phi_{2 i}^{\prime}(t), q_{k} \frac{\partial \Phi_{2 i}(t)}{\partial x_{k}}\right)\right|_{0} ^{T}+\frac{1}{2} \int_{Q} \frac{\partial q_{k}}{\partial x_{k}}\left(\left|\Phi_{2}^{\prime}\right|^{2}-\left|\nabla \Phi_{2}\right|^{2}\right) d x d t \\
& +\int_{Q} \frac{\partial q_{k}}{\partial x_{j}} \frac{\partial \Phi_{2 i}}{\partial x_{j}} \frac{\partial \Phi_{2 i}}{\partial x_{k}} d x d t
\end{aligned}
$$

where $\nabla_{\sigma} \Phi_{2}$ denotes the tangential gradient of $\Phi_{2}$.

Let $\lambda_{0}^{2}>0$ be the first eigenvalues of the following spectral problem

$$
\mid \begin{array}{lll}
-\Delta \Phi=\lambda^{2} \Phi & \text { in } & \Omega \\
\operatorname{div} \Phi=0 & \text { in } & \Omega \\
\frac{\partial \Phi}{\partial \nu}=0 & \text { on } & \Gamma_{0} \\
\Phi=0 & \text { on } & \Gamma_{1}
\end{array}
$$

Lemma 3.4. Assume $T>2 R\left(x_{0}\right)+\frac{(n-1)}{\lambda_{0}}$. Then every solution of the (3.4) with data $\left\{\Phi_{2}^{0}, \Phi_{2}^{1}\right\} \in Y \times X$ verifies

$$
E_{02} \leq \frac{R\left(x_{0}\right)}{2\left(T-2 R\left(x_{0}\right)-\frac{n-1}{\lambda_{0}}\right)} \int_{\Sigma_{0}}\left|\Phi_{2}^{\prime}\right|^{2} d \Sigma .
$$

The proof of the Lemmas 3.3 and 3.4 were done as in Lions [6] adapted to (3.5).

Theorem 3.1. Let $\Omega$ be a domain satisfying (2.1) - (2.3) and $T>4 R\left(x_{0}\right)+\frac{n-1}{\lambda_{0}}$. Then for every data,

$$
\left\{\Phi_{1}^{0}, \Phi_{1}^{1}\right\} \in V \times H, \quad\left\{\Phi_{2}^{0}, \Phi_{2}^{1}\right\} \in Y \times X,
$$

the solutions $\Phi_{1}, \Phi_{2}$ of the homogeneous system 


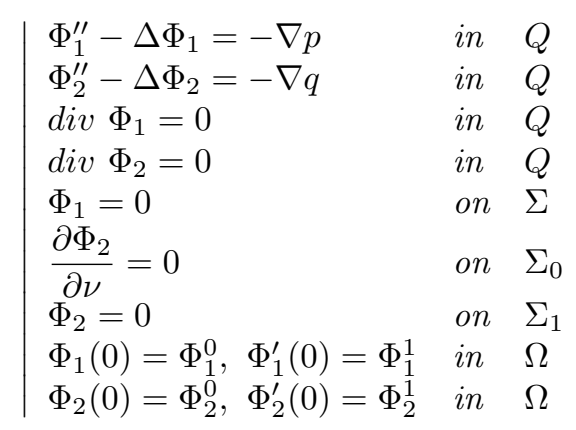

verify

$$
E_{0} \leq \frac{R\left(x_{0}\right)}{2\left(T-T\left(x_{0}\right)\right)} \int_{\Sigma_{0}}\left(\frac{\partial \Phi_{1}}{\partial \nu}+\Phi_{2}^{\prime}\right)^{2} d \Sigma
$$

Proof. From (3.3) and (3.7) we obtain for $T>2 R\left(x_{0}\right)+\frac{n-1}{\lambda_{0}}$,

$$
E_{0} \leq \frac{R\left(x_{0}\right)}{2\left(T-2 R\left(x_{0}\right)-\frac{n-1}{\lambda_{0}}\right)} \int_{\Sigma_{0}}\left(\left|\frac{\partial \Phi_{1}}{\partial \nu}\right|^{2}+\left|\Phi_{2}^{\prime}\right|^{2}\right) d \Sigma .
$$

Suppose that the following inequality is verified

$$
\left|\int_{\Sigma_{0}} \frac{\partial \Phi_{1}}{\partial \nu} \Phi_{2}^{\prime} d \Sigma\right| \leq 2 E_{0}
$$

Then from (3.10) and (3.11) it follows

$$
E_{0} \leq \frac{R\left(x_{0}\right)}{2\left(T-4 R\left(x_{0}\right)-\frac{n-1}{\lambda_{0}}\right)} \int_{\Sigma_{0}}\left(\frac{\partial \Phi_{1}}{\partial \nu}+\Phi_{2}^{\prime}\right)^{2} d \Sigma,
$$

for $T>4 R\left(x_{0}\right)+\frac{n-1}{\lambda_{0}}$.

To conclude the proof of the theorem, it remains then to verify that (3.11) happens.

In fact, multiplying the equation in $(3.8)_{1}$ by $\Phi_{2}^{\prime}$, and integrating on $Q$, we obtain

$$
\int_{Q}\left(\Phi_{1}^{\prime \prime} \Phi_{2}^{\prime}+\nabla \Phi_{1} \nabla \Phi_{2}^{\prime}\right) d x d t-\int_{\Sigma} \frac{\partial \Phi_{1}}{\partial \nu} \Phi_{2}^{\prime} d \Sigma=\int_{Q} p d i v \Phi_{2}^{\prime} d x d t-\int_{\Sigma} p \Phi_{2}^{\prime} d \Sigma
$$

Since $\Phi_{2} \in C([0, T], Y) \cap C^{1}([0, T], X), p$ satisfies $(2.4)$ and $\Phi_{2}=0$ on $\Sigma_{1}$, then from (3.13), it follows

$$
\int_{Q}\left(\Phi_{1}^{\prime \prime} \Phi_{2}^{\prime}+\nabla \Phi_{1} \nabla \Phi_{2}^{\prime}\right) d x d t=\int_{\Sigma_{0}} \frac{\partial \Phi_{1}}{\partial \nu} \Phi_{2}^{\prime} d \Sigma
$$


Now, multiplying the equation $(3.8)_{2}$ by $\Phi_{1}^{\prime}$, and integrating in $Q$, we get

$$
\int_{Q}\left(\Phi_{2}^{\prime \prime} \Phi_{1}^{\prime}+\nabla \Phi_{2} \nabla \Phi_{1}^{\prime}\right) d x d t=\int_{Q} q d i v \Phi_{1}^{\prime} d x d t-\int_{\Sigma} q \Phi_{1}^{\prime} d \Sigma
$$

Since $\Phi_{1} \in C([0, T], V) \cap C^{1}([0, T], H)$, then

$$
\int_{Q}\left(\Phi_{2}^{\prime \prime} \Phi_{1}^{\prime}+\nabla \Phi_{2} \nabla \Phi_{1}^{\prime}\right) d x d t=0
$$

Adding (3.14) and (3.15) comes

$$
\int_{Q} \frac{d}{d t}\left(\Phi_{1}^{\prime} \Phi_{2}^{\prime}+\nabla \Phi_{1} \nabla \Phi_{2}\right) d t d x=\int_{\Sigma_{0}} \frac{\partial \Phi_{1}}{\partial \nu} \Phi_{2}^{\prime} d \Sigma
$$

that is,

$$
\left.\left\{\left(\Phi_{1}^{\prime}(t), \Phi_{2}^{\prime}(t)\right)+\left(\nabla \Phi_{1}(t), \nabla \Phi_{2}(t)\right)\right\}\right|_{0} ^{T}=\int_{\Sigma_{0}} \frac{\partial \Phi_{1}}{\partial \nu} \Phi_{2}^{\prime} d \Sigma
$$

Therefore,

$$
\left|\int_{\Sigma_{0}} \frac{\partial \Phi_{1}}{\partial \nu} \Phi_{2}^{\prime} d \Sigma\right| \leq 2\left(E_{01}+E_{02}\right)=2 E_{0}
$$

concluding the result.

Corollary 3.1. Assume $\Omega$ as in the Theorem 3.1 and $T>4 R\left(x_{0}\right)+\frac{n-1}{\lambda_{0}}$. Let $\Phi_{1}$ and $\Phi_{2}$ be two solutions corresponding to the initial data $\left\{\Phi_{1}^{0}, \Phi_{1}^{1}\right\} \in V \times H$ and $\left\{\Phi_{2}^{0}, \Phi_{2}^{1}\right\} \in Y \times X$ respectively. If $\Phi_{1}$ and $\Phi_{2}$ satisfy

$$
\frac{\partial \Phi_{1}}{\partial \nu}+\Phi_{2}^{\prime}=0 \quad \text { on } \quad \Sigma_{0}, \quad \text { then } \quad \Phi_{1}=\Phi_{2}=0 \quad \text { in } \quad Q .
$$

Proof. The proof follows immediately from (3.9).

\section{Simultaneous Controllability}

The main result of this work is the following theorem:

Theorem 4.2. Let $\Omega$ be a bounded domain of the $\mathbb{R}^{n}, n \geq 2$, satisfying (2.1)-(2.3) and $T>4 R\left(x_{0}\right)+\frac{n-1}{\lambda_{0}}$. Then for every data $\left\{y_{1}^{0}, y_{1}^{1}, y_{2}^{0}, y_{2}^{1}\right\} \in H^{\prime} \times V^{\prime} \times\left(L^{2}(\Omega)\right)^{n} \times X^{\prime}$, there exists a control $v \in\left(L^{2}\left(\Sigma_{0}\right)\right)^{n}$, 
such that the solution $\left\{y_{1}, y_{2}\right\}$ of the system

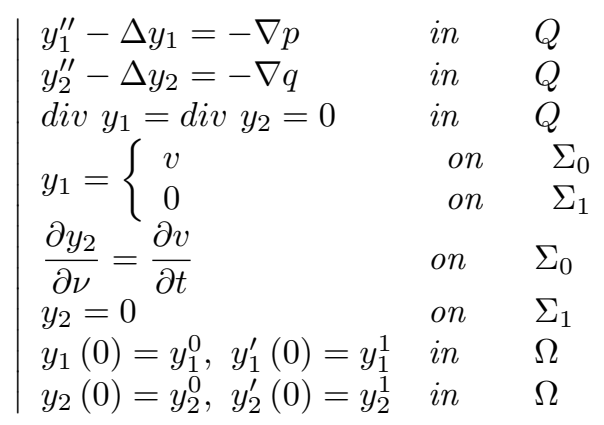

verifies

$$
y_{1}(T)=y_{1}^{\prime}(T)=y_{2}(T)=y_{2}^{\prime}(T)=0 .
$$

Proof. We will apply here the HUM.

First we solve the homogeneous system (3.8) with the initial conditions $\left\{\Phi_{1}^{0}, \Phi_{1}^{1}, \Phi_{2}^{0}, \Phi_{2}^{1}\right\} \in \mathcal{V} \times \mathcal{V} \times Y \times X$

Let us define the quadratic form

$$
\left\|\left\{\Phi_{1}^{0}, \Phi_{1}^{1}, \Phi_{2}^{0}, \Phi_{2}^{1}\right\}\right\|_{F}:=\left\{\int_{\Sigma_{0}}\left|\frac{\partial \Phi_{1}}{\partial \nu}+\Phi_{2}^{\prime}\right|^{2}\right\}^{\frac{1}{2}} .
$$

It follows from the Corollary 3.1, that (4.2) defines a norm in $\mathcal{V} \times \mathcal{V} \times Y \times X$.

We build the space,

$$
F=\overline{\mathcal{V} \times \mathcal{V} \times Y \times X} \|^{\|\cdot\|_{F}} .
$$

From (3.12) follows the immersion

$$
F \hookrightarrow V \times H \times X \times\left(L^{2}(\Omega)\right)^{n} .
$$

Therefore

$$
V^{\prime} \times H^{\prime} \times X^{\prime} \times\left(L^{2}(\Omega)\right)^{n} \hookrightarrow F^{\prime}
$$

with continuous immersion.

Note that

$$
\left\{\Phi_{1}^{0}, \Phi_{1}^{1}, \Phi_{2}^{0}, \Phi_{2}^{1}\right\} \in F \Leftrightarrow \frac{\partial \Phi_{1}}{\partial \nu}+\Phi_{2}^{\prime} \in\left(L^{2}\left(\Sigma_{0}\right)\right)^{n} .
$$

On the other hand, from (3.3), the continuity of the application (3.2) and (4.3), we obtain

$$
\left\{\Phi_{1}^{0}, \Phi_{1}^{1}\right\} \in V \times H \Leftrightarrow \frac{\partial \Phi_{1}}{\partial \nu} \in\left(L^{2}\left(\Sigma_{0}\right)\right)^{2} .
$$

Hence, from (4.4) and (4.5), it follows that

$$
\left\{\Phi_{1}^{0}, \Phi_{1}^{1}, \Phi_{2}^{0}, \Phi_{2}^{1}\right\} \in F \Leftrightarrow\left\{\begin{array}{c}
\left\{\Phi_{1}^{0}, \Phi_{1}^{1}\right\} \in V \times H \\
\text { and } \\
\Phi_{2}^{\prime} \in\left(L^{2}\left(\Sigma_{0}\right)\right)^{n} .
\end{array}\right.
$$


Thus, we consider the norm

$$
\left\|\left\{\Phi_{2}^{0}, \Phi_{2}^{1}\right\}\right\|_{G}:=\left\{\int_{\Sigma_{0}}\left|\Phi_{2}^{\prime}\right|^{2} d \Sigma\right\}^{\frac{1}{2}},
$$

and the Hilbert space

$$
G=\overline{Y \times X} \|^{\|\cdot\|_{G}} .
$$

From (3.7), it follows

$$
G \hookrightarrow X \times\left(L^{2}(\Omega)\right)^{n}
$$

Therefore,

$$
F=V \times H \times G
$$

and

$$
F^{\prime}=V^{\prime} \times H^{\prime} \times G^{\prime}
$$

We consider the following backward system

$$
\begin{array}{ll}
\Psi_{1}^{\prime \prime}-\Delta \Psi_{1}=-\nabla p & \text { in } Q \\
\Psi_{2}^{\prime \prime}-\Delta \Psi_{2}=-\nabla q & \text { in } Q \\
\operatorname{div} \Psi_{1}=\operatorname{div} \Psi_{2}=0 & \text { in } Q \\
\Psi_{1}=\frac{\partial \Phi_{1}}{\partial \nu}+\Phi_{2}^{\prime} & \text { on } \Sigma_{0} \\
\Psi_{1}=0 & \text { on } \Sigma_{1} \\
\frac{\partial \Psi_{2}}{\partial \nu}=\frac{\partial}{\partial t}\left(\frac{\partial \Phi_{1}}{\partial \nu}+\Phi_{2}^{\prime}\right) & \text { on } \Sigma_{0} \\
\Psi_{2}=0 & \text { on } \Sigma_{1} \\
\Psi_{1}(T)=\Psi_{1}^{\prime}(T)=\Psi_{2}(T)=\Psi_{2}^{\prime}(T)=0 & \text { in } \Omega,
\end{array}
$$

where $\frac{\partial}{\partial t}\left(\frac{\partial \Phi_{1}}{\partial \nu}+\Phi_{2}^{\prime}\right)$ is taken in the following sense

$$
\left\langle\frac{\partial}{\partial t}\left(\frac{\partial \Phi_{1}}{\partial \nu}+\Phi_{2}^{\prime}\right), v\right\rangle=-\int_{\Sigma_{0}}\left(\frac{\partial \Phi_{1}}{\partial \nu}+\Phi_{2}^{\prime}\right) v^{\prime} d \Sigma,
$$

for all $v \in H_{0}^{1}\left(0, T ;\left(L^{2}\left(\Gamma_{0}\right)\right)^{n}\right)$.

Consider now the application

$$
\Lambda: F \rightarrow F^{\prime},
$$

defined by

$$
\Lambda\left\{\Phi_{1}^{0}, \Phi_{1}^{1}, \Phi_{2}^{0}, \Phi_{2}^{1}\right\}=\left\{\Psi_{1}^{\prime}(0),-\Psi_{1}(0), \Psi_{2}^{\prime}(0),-\Psi_{2}(0)\right\},
$$

where $\left\{\Psi_{1}, \Psi_{2}\right\}$ is the solution of (4.6).

The norm in (4.2) induces in $\mathcal{V} \times \mathcal{V} \times Y \times X$ the following inner product

$$
\left\langle\left\{\Phi_{1}^{0}, \Phi_{1}^{1}, \Phi_{2}^{0}, \Phi_{2}^{1}\right\},\left\{\xi_{1}^{0}, \xi_{1}^{1}, \xi_{2}^{0}, \xi_{2}^{1}\right\}\right\rangle_{F}=\int_{\Sigma_{0}}\left(\frac{\partial \Phi_{1}}{\partial \nu}+\Phi_{2}^{\prime}\right)\left(\frac{\partial \xi_{1}}{\partial \nu}+\xi_{2}^{\prime}\right) d \Sigma,
$$


hence

$$
\left\langle\Lambda\left\{\Phi_{1}^{0}, \Phi_{1}^{1}, \Phi_{2}^{0}, \Phi_{2}^{1}\right\},\left\{\Phi_{1}^{0}, \Phi_{1}^{1}, \Phi_{2}^{0}, \Phi_{2}^{1}\right\}\right\rangle_{F}=\left\|\left\{\Phi_{1}^{0}, \Phi_{1}^{1}, \Phi_{2}^{0}, \Phi_{2}^{1}\right\}\right\|_{F}^{2}
$$

and $\Lambda$ is a isomorphism between $F$ and $F^{\prime}$. Therefore, for every $\left\{y_{1}^{0}, y_{1}^{1}, y_{2}^{0}, y_{2}^{1}\right\} \in F^{\prime}$, there exists only one $\left\{\Phi_{1}^{0}, \Phi_{1}^{1}, \Phi_{2}^{0}, \Phi_{2}^{1}\right\} \in F$ such that

$$
\Lambda\left\{\Phi_{1}^{0}, \Phi_{1}^{1}, \Phi_{2}^{0}, \Phi_{2}^{1}\right\}=\left\{y_{1}^{1},-y_{1}^{0}, y_{2}^{1},-y_{2}^{0}\right\}
$$

By (4.7) and (4.8) we conclude that the unique ultra weak solution of (4.6) satisfies

(1.1). Then the unique ultra weak solution of (1.1), with control $v=\frac{\partial \Phi_{1}}{\partial \nu}+\Phi_{2}^{\prime}$ verifies (1.2).

Acknowledgement. We wish to acknowledge the Referees of TEMA (Tendências em Matemática Aplicada e Computacional ) for their constructive remarks and corrections in the manuscript.

Resumo. Neste trabalho estudamos a controlabilidade simultânea para um sistema de equações que representam um modelo da dinâmica de elasticidade para materiais imcompressíveis.

\section{References}

[1] H. Brezis, "Analyse Fonctionelle, Théorie et Applications", Dunod, Paris, 1999

[2] M.M. Cavalcanti, V.N. Domingos Cavalcanti, A. Rocha and J.A. Soriano, Exact controllability of a second-order integro-differential equation with a pressure term, EJQTDE, 9 (1998), 1-18.

[3] A. Haraux, On a completion problem in the theory of distribute control of wave equations, "Nonlinear Partial Differencial Equations and Their Applications", Lect. Collège de France Seminar, Paris 1987-88, Vol. X, Pitman Reserch Notes in Math., 220 (1991), 242-271.

[4] B. Kapitonov, "Simultaneous Observability of Some Hyperbolic Systems", relatório de pesquisa e desenvolvimento, LNCC.

[5] V. Komornik, "Controllability and Stabilization. The Multiplier Method", Masson, Paris, 1994.

[6] J.L. Lions, "Exact Controllability, Stabilization and Pertubations for Distributed Systems", J. Von Newmann Lecture, Boston, 1986, SIAM Review, March, 1988.

[7] J. L. Lions, On some hyperbolic equations with a pressure term, em "Proceedings of the conference dedicated to Louis Nirenberg", Trento, Italy, september 3-8, 1990, Harlow: Longman Scientific and Technical, Pitman Res. Notes Math. Ser., 269 ( 1992), 196-208.

[8] E. Zuazua, Contrôle simultané de deux équations des ondes, to appear. 